\title{
Computational analysis of deformation and fracture in composite materials and coatings
}

\author{
Ruslan Balokhonov ${ }^{1, *}$, Rustam Bakeev ${ }^{1}$, Varvara Romanova ${ }^{1}$, Evgenia Emelianova $^{1}$, \\ Maksim Sergeev ${ }^{1}$, Aleksandr Zinoviev ${ }^{1}$, Olga Zinovieva ${ }^{1}$, Ekaterina Batukhtina ${ }^{1}$, and \\ Eugene Schwab ${ }^{2}$ \\ ${ }^{1}$ Institute of Strength Physics and Materials Science, SB, RAS, 634055 Tomsk, Russia \\ ${ }^{2}$ Doppelmayer Seilbahnen GmbH, 6922 Wolfurt, Austria
}

\begin{abstract}
Deformation and fracture of metal-ceramic composites and coatings under quasistatic tension, compression, shear, and during cooling from the melt to room temperatures are numerically investigated. Dynamic and quasistatic boundary-value problems are solved in two- and threedimensional formulations by the finite-difference and finite-element methods. Constitutive models include the isotropic hardening of aluminum and fracture of ceramic particles. Composite microstructure is taken into account explicitly. Analysis of the fracture in composites is carried out, with the residual stresses being not taken into consideration. Origination of cracks in the near-interface regions of bulk tension and their propagation in ceramic particles are investigated. Residual stresses formed during cooling of the composites are calculated for different volume fractions of particles.
\end{abstract}

\section{Introduction}

Nowadays there is an intensive experimental investigation of metal-matrix composites and coatings (MMC) fabricated by different deposition techniques, for instance, selective laser melting [1] and solid-state sintering [2]. The composite materials possess strongly inhomogeneous microstructure, with the irregular interfaces being observed (Fig. 1). Microstructural inhomogeneity including pores, reinforcing particles, polycrystalline grains, coatings, initial cracks, etc. is the source of stress-strain concentration [3-12]. Changes in microstructure characteristics are found to give rise to the changes in the strength and durability of the composites. Effects of the reinforcing particle size and weight/volume fraction on the mechanical properties of the composite coatings are analyzed [3]. Volume fraction of carbide particle of $20 \%$ is shown to be optimal, as the high residual porosity is formed at higher values of the fraction.

The most of microstructure-based numerical simulation of MMC deformation deals with idealized round- or spherically-shaped particles.

The aim of this paper is to study interrelated processes of plastic strain localization in the metal matrix and cracking in ceramic particles, as well as appearance of residual stresses in the MMC subjected to mechanical and thermal loadings.

\footnotetext{
* Corresponding author: rusy@ispms.tsc.ru
} 


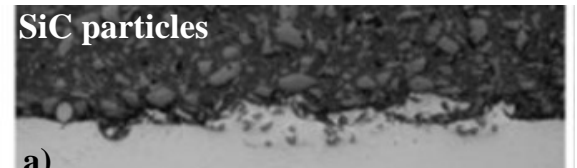

a)

AA6082 matrix/substrate

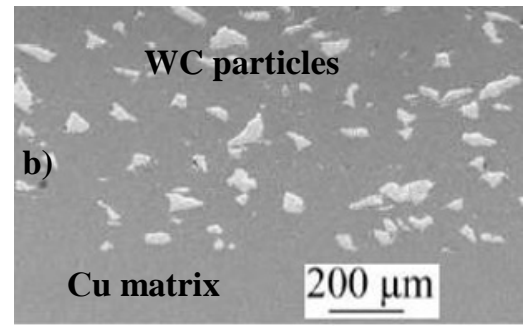

Fig. 1. Microstructure of "metal matrix - carbide particles" composite coatings: a) [1], b) [2].

\section{Deformation and fracture under mechanical loading}

Detailed 3D analysis of stress-strain localization in a carbide particle embedded into the aluminum microvolume subjected to simple uniaxial compression or shear was carried out by ABAQUS Standard. Dangerous regions of bulk tension are shown to be formed, in which cracks may arise as the loading continues (Fig. 2b). Extended local regions of positive cubic strains are found even under pure shear, which is characterized by the absence of cubic strains at the macro level at all. Moreover, these regions formed under shear are even more pronounced, and the stress concentration in these regions is higher than under compression. Thus, it has been shown that pure shearing initiates local bulk tension in composites more intensive than compression.
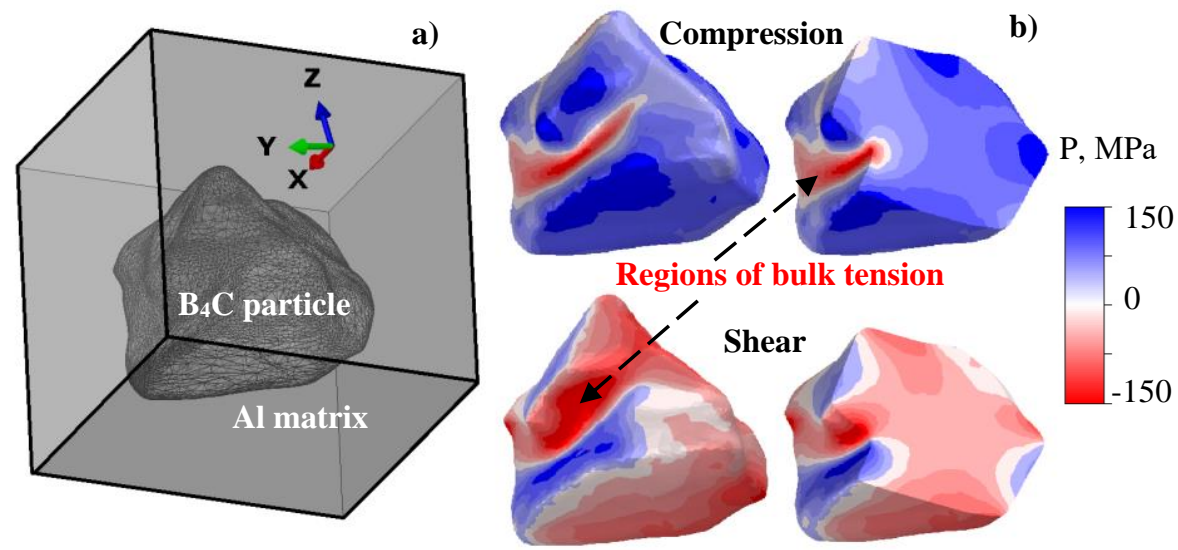

Fig. 2. Microvolume with single particle (a) and formation of local regions of bulk tension under compression and pure shear of the composite (b). Volume fraction of the particle is $20 \%$.

Crack origination in the regions of bulk tension was simulated in two-dimensions (Fig. 3). Dynamic boundary-value problem was solved by the finite-difference method. Cracks under external tension or compression originate in tensile regions located in different sites of the particle and propagate under the action of local tensile loads in different directions along and perpendicular to the loading direction, respectively (Fig. 3a), which fully agrees with the experimental data. Under tension of the coated material, cracks propagate perpendicular to the loading direction that unloads the coating in this direction. Therefore, through-the-thickness cracks are formed in the coating, propagating toward the free surface and "coating-substrate" interface. As a crack reaches the interface, an additional stress concentration from the crack tip initiates macroscopic localized plastic deformation bands in the aluminum substrate (Fig. 3b). The transverse cracks formed in thick coatings under tension decreases the macroscopic strength of the coated material. During compression, 
multiple cracking of the coating is realized. Cracks successively originate in particles and propagate along the loading direction. In this case, unloading along the direction of compression does not occur. Therefore, the coating cracks evenly throughout the volume.

a)

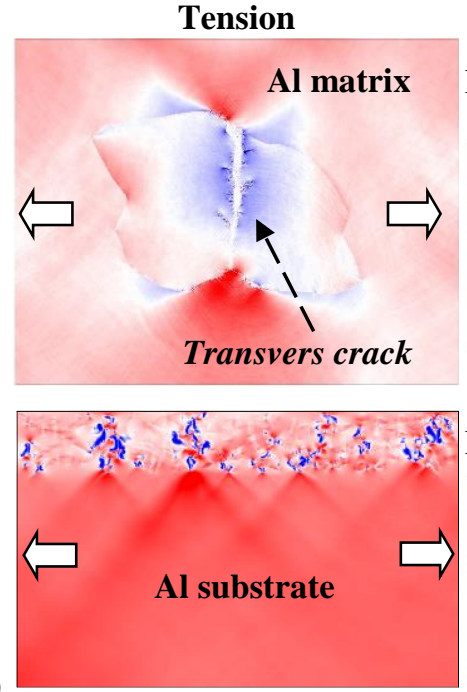

b)

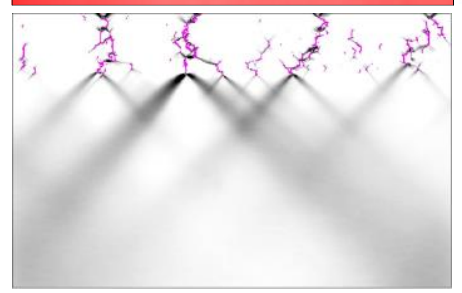

Compression
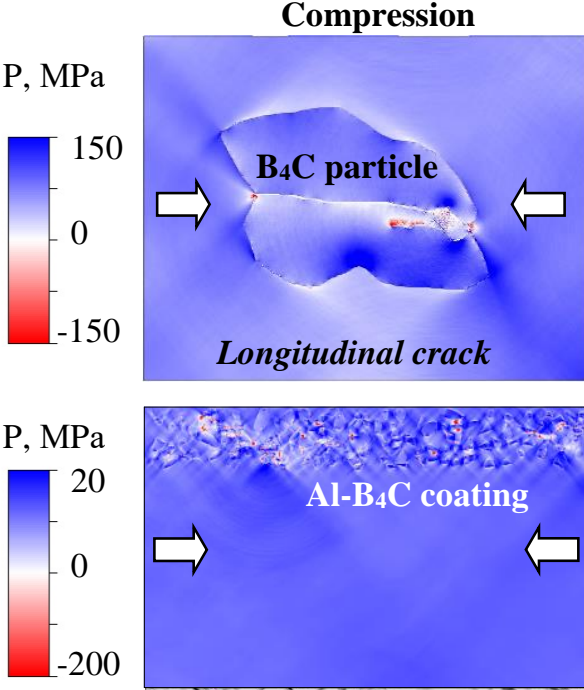

$\mathrm{P}, \mathrm{MPa}$
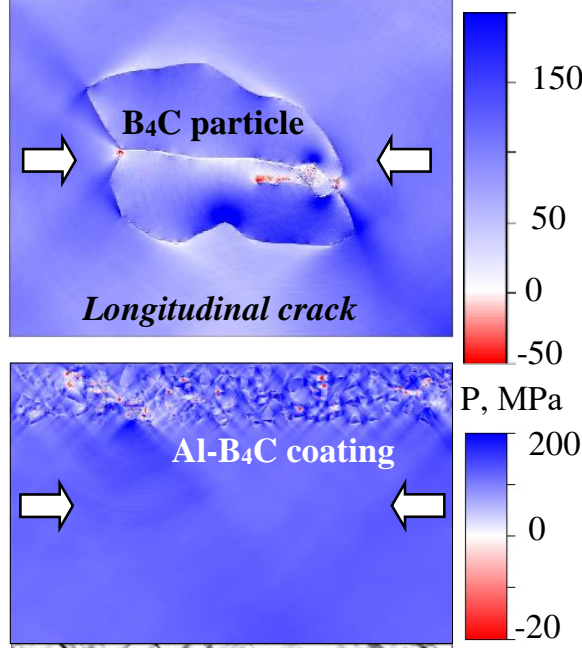
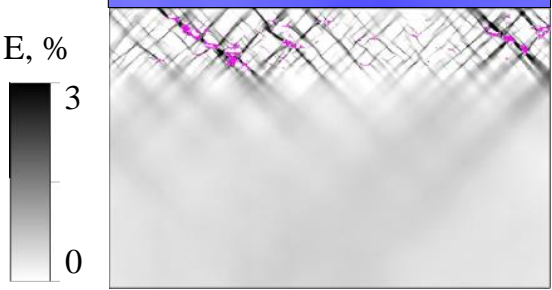

Fig. 3. Crack propagation in single particle (a) and in composite coating (b) under tension and compression of the composite materials. $\mathrm{P}$ is the pressure and $\mathrm{E}$ is the equivalent plastic strain with the fractured regions marked by magenta color.

\section{Residual stresses under cooling}

Formation of residual stresses and the influence of the particle size on the stress concentration and plastic strain localization were investigated during cooling of the MMC from 400 to $23 \mathrm{C}^{\circ}$ that happens after selective laser melting. Calculations were performed for the microstructures shown in Fig. 2a and 4a, containing single and twelve ceramic particles. The volume fraction of the boron carbide was $20 \%$ in both cases. Contrary to the uniaxial compression, where the local tensile regions are formed both in the particle (Fig. 2) and matrix, under bulk compression realized during cooling these regions were found to arise mostly in the aluminum matrix, with only a few particle corners being subjected to tension (Fig.4b).

Compressive cubic strain of the composite after cooling is about $1.5 \%$. The calculation results showed that for the particle volume fraction of $20 \%$ the most of the aluminum matrix experiences plastic deformation, with the residual stresses in the matrix being $30 \mathrm{MPa}$ and higher. Equivalent stress distributions in composites with varied particle size are presented in Fig. 4c. The stress concentrates around the particles, causing the plastic strain localization in the near-interface regions. The smaller is the particle, the larger is the area of the particle surface. Accordingly, the stress concentration and plastic strain localization increases with the particle size decreasing, and the macroscopic residual stress decreases with the particle size. 


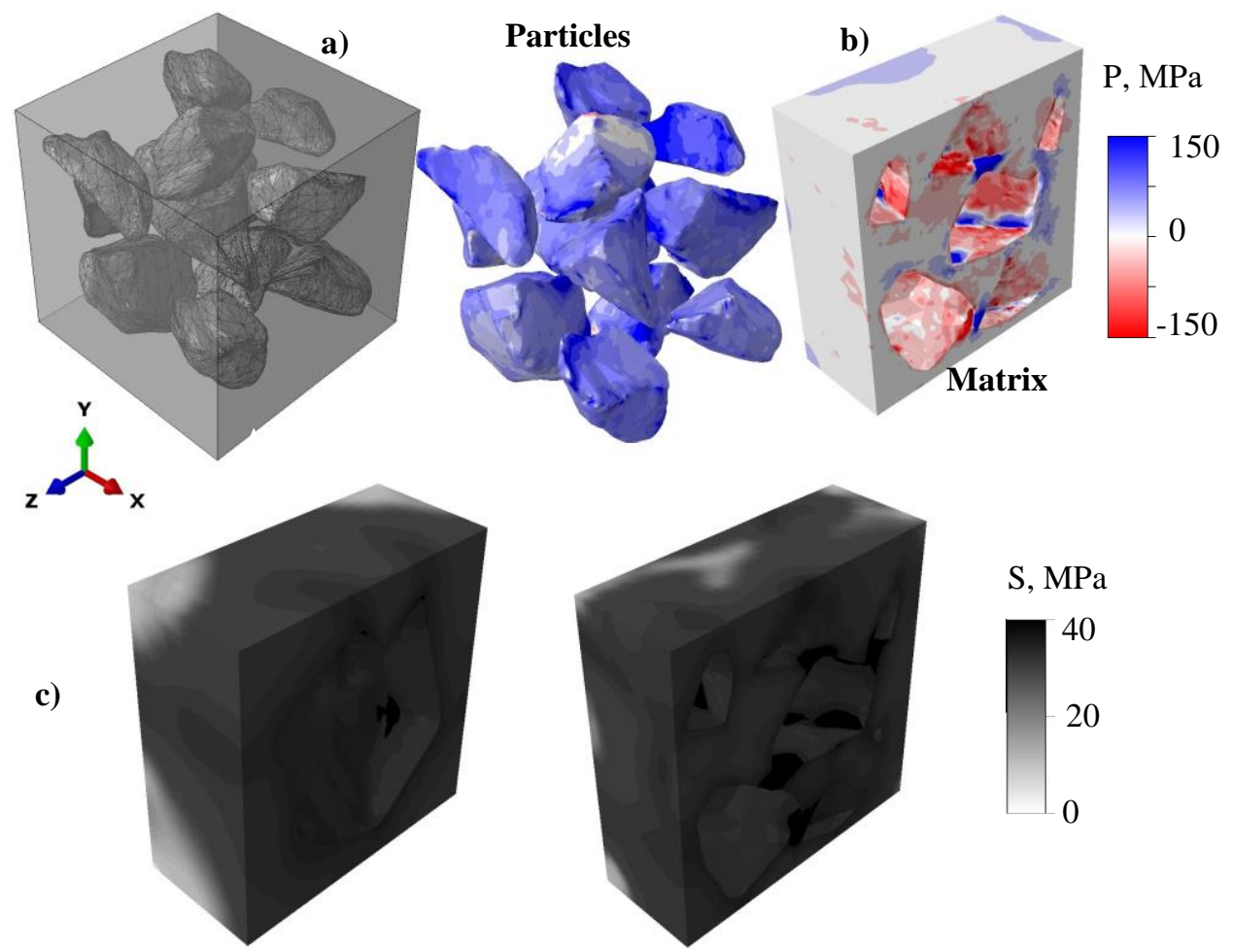

Fig. 4. Microvolume with 12 particles (a) and formation of local regions of bulk tension under cooling of the composite (b). Volume fraction of particles is $20 \%$. Equivalent stress patterns (c) in the aluminium matrix for microstructures shown in Figs. 2a and 4a.

The work is supported by Russian Science Foundation (Project No. 18-19-00273).

\section{References}

1. A. Riquelme, M.D. Escalera-Rodríguez, P. Rodrigo et al., Journal of Alloys and Compounds, 727 671-682 (2017).

2. J.L. Cabezas-Villa, L. Olmos, H.J. Vergara-Hernandez et al., Trans. Nonferrous Met. Soc. China, 27 2214-2224 (2017).

3. I.S. Konovalenko, A.I. Dmitriev, A.Y. Smolin, et al., Phys. Mesomech., 15 1-2 88-93 (2012).

4. R.R.Balokhonov, V.A.Romanova, A.V.Panin, et al., Phys. Mesomech. 211 32-42 (2018).

5. A.Yu. Smolin, Ig.S.Konovalenko, S.N Kul'kov, et al., Tech. Phys. Let., 32 9 738-740 (2006).

6. I.Yu. Smolin, P.V. Makarov, M.O. Eremin, et al., Proc. Struct. Integr., 2 3353-3360 (2016).

7. O.I. Cherepanov, I.Yu. Smolin, Yu.P. Stefanov, et al., Comput. Mater. Sci., 16 25-31 (1999).

8. A.Yu. Smolin, E. Shilko, S. Astafurov, et al., Defence Tech., 14 643-656 (2018).

9. A.I. Dmitriev, A.Yu. Smolin, V.L. Popov, et al., Phys. Mesomech., 12 11-19 (2009).

10. A.I. Dmitriev, A.Yu. Smolin, S.G. Psakhie, et al., Phys. Mesomech., 11 73-84 (2008).

11. S.G.Psakhie, E.V.Shilko, S.V.Astafurov, Tech. Phys. Let., 30 237-239 (2004).

12. S.G.Psakhie, E.V.Shilko, M.V.Popov, et al., Phys. Rev. E, 91063302 (2015). 